\title{
Suppression of the p75 receptor signal attenuates the effect of ephrin-B3 and promotes axonal regeneration of the injured optic nerve
}

\author{
N Uesugi ${ }^{1,2}$, Y Kimura ${ }^{1,2}$ and T Yamashita ${ }^{\star, 1,2}$
}

The p75 neurotrophin receptor (p75NTR) is known to transduce the signal from some myelin-associated axon growth inhibitors, including Nogo and myelin-associated glycoprotein. As ephrin-B3, a member of the ephrin family, is also expressed in myelin and inhibits axon growth, the purpose of this study was to assess the possible involvement of p75NTR in ephrin-B3 signaling. Here, we report that p75NTR is required for the inhibitory effect of ephrin-B3 on neurite growth in vitro. While ephrin-B3 inhibited neurite elongation of embryonic cortical neurons, the neurons with p75NTR knockdown or with EphA4 knockdown were less sensitive to ephrin-B3. Although no direct interaction of p75NTR with ephrin-B3 was observed, Pep5, a peptide that specifically inhibits RhoA activation mediated by p75NTR, reduced the effect of ephrin-B3. Therefore, p75NTR functions as a signal transducer for ephrin-B3. Moreover, axonal regeneration in vivo was induced by Pep5 application after optic nerve crush injury in mice. Thus, Pep5 is a promising agent that contributes to axonal regeneration in the central nervous system.

Cell Death and Disease (2013) 4, e557; doi:10.1038/cddis.2013.83; published online 21 March 2013

Subject Category: Experimental Medicine

Axons in the adult mammalian central nervous system (CNS) fail to regenerate after injury due to the presence of an inhibitory environment, which can be partially attributed to molecules expressed in CNS myelin, including myelinassociated glycoprotein (MAG), Nogo, and oligodendrocyte myelin glycoprotein (OMgp). ${ }^{1}$ A receptor complex for these proteins includes the Nogo receptor, p75 neurotrophin receptor (p75NTR) or Troy, and LINGO-1. Downstream of this receptor complex, RhoA has a key role in inhibiting axon growth. In elongating axons, neuronal RhoA is captured by Rho-GDP dissociation inhibitor (Rho-GDI) and activation by intracellular Rho guanine nucleotide exchange factors (RhoGEFs) is prevented. In response to ligand binding to the receptor complex, p75NTR facilitates dissociation of Rho-GDI from $R$ hoA, ${ }^{2}$ and the released GDP-bound form of RhoA is converted into the GTP-bound form by Kalirin9, an intracellular Rho-GEF. ${ }^{3}$ As neurons from p75NTR-deficient mice exhibit lower sensitivity to myelin, ${ }^{4}$ p75NTR was considered a critical molecule for axonal growth inhibition after injury. However, several reports have shown that little CNS axonal regeneration was observed in p75NTR-deficient mice., Interpreting these results is complicated, as p75NTR transduces bidirectional signals when regulating axonal growth. Neurotrophin binding to p75NTR promotes axonal elongation via inhibition of $\mathrm{RhoA}^{7}$ whereas RhoA activation through
p75NTR was elicited by MAG, Nogo, and OMgp. ${ }^{4,8}$ Indeed, p75NTR-deficient mice show retarded outgrowth of peripheral axons during development. ${ }^{7}$ Individual interpretation of the bidirectional p75NTR signals is required for clearly understanding the role of p75NTR, especially in vivo. Moreover, the ability of semaphorin $3 \mathrm{~A}$, and other semaphorin family members, to collapse growth cones is suppressed in p75NTR-deficient mice, ${ }^{9}$ further supporting the role of p75NTR in axon growth inhibition. In this study, a previously characterized peptide, Pep5, was used to interpret p75NTR signals. $^{2}$ Pep5 is a synthetic peptide derived from the intracellular domain of p75NTR and specifically inhibits the interaction of Rho-GDI with p75NTR, making Pep5 an appropriate tool for understanding the role of p75NTR in CNS axonal growth inhibition.

The first goal of this study was to assess the possible involvement of p75NTR in other myelin-derived proteins by using Pep5. Results showed that Pep5 also inhibited the effect of ephrin-B3, a member of the ephrin family. Ephrin-B3 is expressed in oligodendrocytes in the spinal cord and inhibits axonal elongation in vitro. ${ }^{10}$ For example, regeneration of the optic nerve and corticospinal axons was enhanced in ephrinB3-deficient mice following their injuries. ${ }^{11}$ Indeed, this study demonstrated that $\mathrm{p} 75 \mathrm{NTR}$ has a role in signal transduction from ephrin-B3. Therefore, p75NTR mediates the effects of

\footnotetext{
${ }^{1}$ Department of Molecular Neuroscience, Graduate School of Medicine, Osaka University, 2-2 Yamadaoka, Suita, Osaka, Japan and ${ }^{2}$ Core Research for Evolutional Science and Technology (CREST), Japan Science and Technology Agency (JST), 5, Sanbancho, Chiyoda-ku, Tokyo, Japan

${ }^{*}$ Corresponding author: T Yamashita, Department of Molecular Neuroscience, Graduate School of Medicine, Osaka University, 2-2 Yamadaoka, Suita, Osaka 565-0871, Japan. Tel.: + 8166879 3661; Fax: + 8166879 3669; E-mail: yamashita@molneu.med.osaka-u.ac.jp

Keywords: optic nerve injury; ephrin; p75; regeneration; axon; myelin

Abbreviations: CNS, central nervous system; p75NTR, p75 neurotrophin receptor; Rho-GDI, Rho-GDP dissociation inhibitor; Rho-GEFs, Rho guanine nucleotide exchange factors; GTP, guanosine triphosphate; GDP, guanosine diphosphate; ECD, extracellular domain; ELISA, enzyme-linked immunosorbent assay; His, histidine tag; CTB, cholera toxin beta subunit; SDS-PAGE, sodium dodecyl sulphate-polyacrylamide gel electrophoresis; DME/F12, Dulbecco's modified Eagle's medium nutrient mixture F12; DMEM, Dulbecco's modified Eagle's medium; FBS, fetal bovine serum; PBS, phosphate-buffered solution

Received 08.8.12; revised 05.2.13; accepted 20.2.13; Edited by A Verkhratsky
} 
multiple inhibitory molecules expressed in CNS myelin and may provide an efficient molecular target against CNS injury. Further support for this hypothesis was found through the application of Pep5, which enhanced axonal regeneration after optic nerve crush.

\section{Results}

Ephrin-B3 inhibits neurite elongation via RhoA activation. It has previously been reported that ephrin-B3 inhibits neurite extension from postnatal cortical neurons and cerebellar neurons. ${ }^{10}$ Therefore, a neurite outgrowth assay was used to explore the signal transduction of ephrin-B3. Cortical neurons were cultured for $24 \mathrm{~h}$ from mice (postnatal day 1) on confluent monolayers of either $\mathrm{CHO}$ cells expressing mouse ephrin-B3 (ephrin-B3-CHO) or control$\mathrm{CHO}$ cells, and the neurite outgrowth rate was assessed (the co-culture assay). Cortical neurons plated on ephrin-B3-CHO had shorter neurite outgrowth than neurons plated on control-CHO (Figure 1a). Previous studies have shown that ephrins cause axonal repulsion that requires Rho-Rho kinase activity. ${ }^{12}$ In order to determine whether the inhibitory effect observed in this study depends on the Rho-Rho kinase pathway, neurons were cultured for $24 \mathrm{~h}$ on ephrinB3- $\mathrm{CHO}$ cells in the presence of $10 \mu \mathrm{M}$ Y27632, a selective Rho kinase inhibitor. Y27632 partially, but significantly, attenuated the effect of ephrin-B3 (Figures 1a and b). This concentration was known to be effective in blocking Rho kinase activity. These results suggest that ephrin-B3 inhibits neurite growth through Rho activation.

p75NTR and EphA4 are essential for neurite growth inhibition by ephrin-B3. Neurons from p75NTR knockout mice have previously been shown to lose sensitivity to MAG, Nogo-66, and OMgp. ${ }^{2,4}$ In addition, EphA reverse-signaling requires p75NTR for axonal repulsion. ${ }^{13,14}$ Growth cone collapse, induced by Sema-3A and ephrin-B2, was also suppressed in p75NTR-deficient sympathetic neuron. ${ }^{9}$ These findings prompted an examination of the effect of p75NTR knockdown on neurite growth inhibition by ephrinB3. Transfection of p75NTR siRNA in cortical neurons from E18 mice resulted in a reduction of p75NTR expression (Figures 1c and d). These neurons were replated on ephrinB3-expressing $\mathrm{CHO}$ or control-CHO cells. The p75NTR knockdown neurons became less responsive to ephirin-B3 in the neurite growth assay (Figures $1 e$ and $f$ ). The neurons were then transfected with EphA4 siRNA resulting in a reduction in the level of EphA4 expression (Figure $2 a, b$ ). As expected, knockdown of EphA4 led to attenuation of the effect of ephrin-B3 (Figures $2 c$ and d. To examine if RhoA is involved in neurite growth inhibition induced by ephrin-B3, we performed active RhoA pulldown assay. HEK293T cells were transfected with p75NTR and/or EphA4, and were stimulated with $10 \mu \mathrm{g} / \mathrm{ml}$ ephrin-B3 for $5 \mathrm{~min}$. Activation of RhoA was detected only in the cells in which p75NTR and EphA4 were expressed (Figure $2 e$ and $f$ ). These results demonstrate that p75NTR, as well as EphA4, is required for neurite growth inhibition and RhoA activation induced by ephrin-B3 in cortical neurons.
Pep5 reverses the inhibitory effect of ephrin-B3 on neurite elongation. To determine if inhibition of the myelin signal, mediated by p75NTR, attenuated the effects of ephrin-B3 on neurite growth of embryonic cortical neurons, Pep5, which blocks the interaction of p75NTR with RhoGDI, ${ }^{2}$ was employed. Pep5 was fused with a TAT sequence to enable translocation of peptides across the cell membrane (TAT-Pep5). Cortical neurons were cultured for $24 \mathrm{~h}$ on ephrin-B3-CHO in the presence of $1 \mu \mathrm{M}$ TAT-Pep5 or TATfused control peptide. TAT-Pep5, but not TAT-control, reversed the inhibitory effect of ephrin-B3, whereas TATPep5 itself did not modulate neurite length (Figures $3 a$ and b). Moreover, Pep5 treatment inhibited the activation of RhoA induced by ephrin-B3 (Figures $3 c$ and d). As we failed in finding interaction of EphA4 with Rho-GDI (Figure 3e), it is conceivable that Pep5 specifically inhibits the p75NTR signaling in these cells. These findings suggest that ephrinB3 inhibits neurite outgrowth through p75NTR-dependent RhoA activation.

Ephrin-B3 binds indirectly to p75NTR. Multiple ligands, including NGF, BDNF, NT3 and NT4/5, bind to p75NTR. ${ }^{15}$ In order to assess whether ephrin-B3 is a ligand of p75NTR, recombinant proteins of the p75NTR extracellular domain (ECD) and EphA4 ECD were generated. Coomassie Brilliant Blue stain and western blot showed that both proteins were successfully produced (data not shown). ELISA was used to investigate whether ephrin-B3 interacted directly with p75NTR ECD-His and EphA4 ECD-His. Plastic wells were coated with $0.1 \mu \mathrm{g} / \mathrm{ml}$ p75NTR ECD-His or EphA4 ECD-His, and recombinant ephrin-B3-Fc at various concentrations (0$1 \mu \mathrm{g} / \mathrm{ml}$ ) was added. We confirmed binding of recombinant BDNF to p75NTR ECD, demonstrating that the p75NTR ECD proteins were properly folded (Figure $3 f$ ). Although binding of ephrin-B3-Fc to EphA4 ECD-His was detected, no significant binding of ephrin-B3-Fc to p75NTR ECD-His was detected (Figure 3f). Next, a co-precipitation assay was performed using HEK293T cells. These cells did not endogenously express p75NTR or EphA4 (data not shown). The cells were transfected with p75NTR and/or EphA4 and subsequently treated with or without ephrin-B3-Fc. Then, ephrin-B3-Fc was precipitated from the lysates, followed by western blotting. Results revealed that p75NTR co-precipitated with ephrinB3-Fc when EphA4 was expressed, whereas it did not coprecipitate if EphA4 was not expressed (Figure $3 \mathrm{~g}$ ). Therefore, p75NTR binding with ephrin-B3 requires EphA4. These findings strongly suggest that ephrin-B3 binds indirectly to p75NTR via EphA4 and that p75NTR functions as a signal transducer for ephrin-B3.

Pep5 promotes axonal regeneration after optic nerve injury. Oligodendrocytes in the optic nerve express a number of inhibitory molecules, including ephrin-B3. ${ }^{16-18}$ Neurotrophins are upregulated in GCL after optic nerve injury ${ }^{19}$ and both p75NTR and EphA4 are expressed in the optic axons. ${ }^{13,20}$ To assess whether inhibition of p75NTR signaling enhances axonal regeneration in the CNS, an optic nerve crush injury model was used in mice. Optic nerve crush was performed, and TAT-Pep5 or TAT-control peptide was subsequently injected into the eyes of the mice on the same 
a

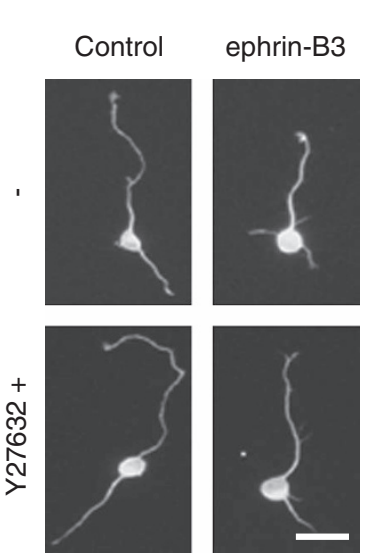

C

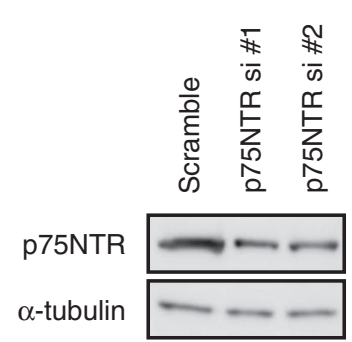

b
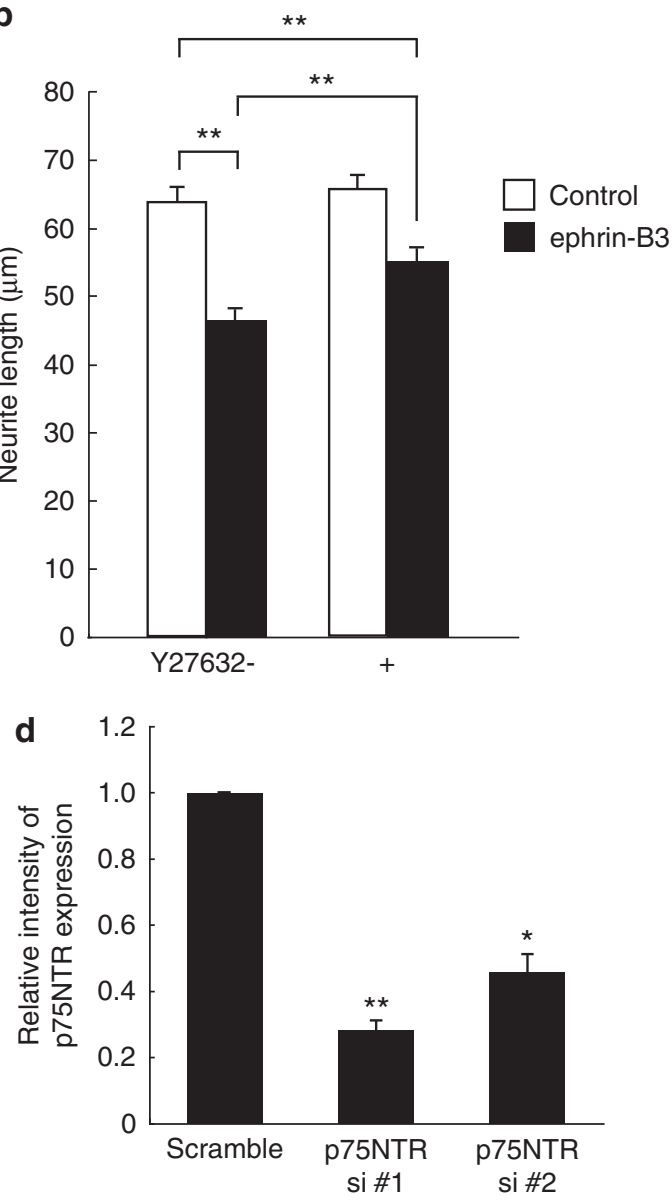

$\mathbf{f}$

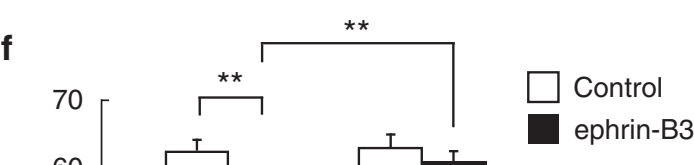

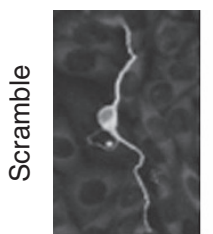

ephrin-B3
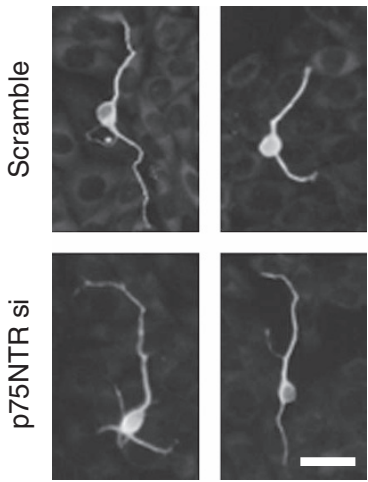

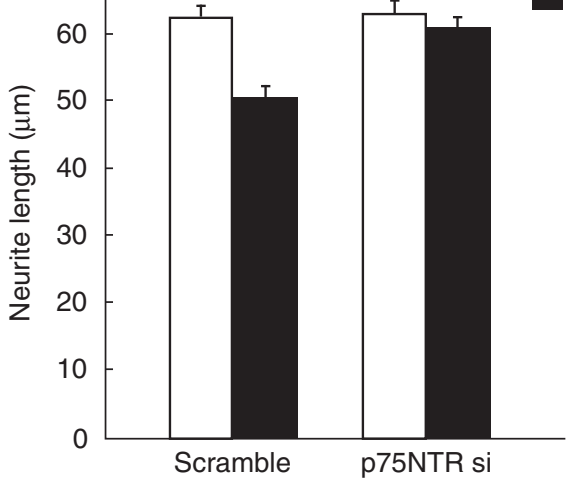

Figure 1 Ephrin-B3 inhibits neurite extension via RhoA activation and p75NTR. (a) The images demonstrate neuronal extension of the neurites in vitro, under the indicated conditions. Cortical neurons were cultured for $24 \mathrm{~h}$ on control/ephrin-B3-expressing CHO cells in the presence or absence of $10 \mu \mathrm{M} \mathrm{Y} 27632$. Scale bar $=20 \mu \mathrm{m}$. (b) The graph illustrates mean neurite length $(n=1064)$. Y27632 significantly attenuated the inhibitory effect of ephrin-B3 on neurite growth. (c) Western blots for p75NTR and $\alpha$-tubulin in p75NTR siRNA- or scramble siRNA-transfected cortical neurons. (d) Quantitative data for $\mathrm{C}(n=3)$. (e) The siRNA-transfected cortical neurons were cultured on control/ ephrin-B3-CHO cells for $24 \mathrm{~h}$. Scale bar $=20 \mu \mathrm{m}$. (f) The graph illustrates mean neurite length $(n=510)$. Knockdown of $p 75 N T R$ blocked neurite growth inhibition induced by ephrin-B3. (b), (d), (f); ${ }^{*} P<0.05,{ }^{* *} P<0.01$, Scheffé's F-test

day (Figure 4a). Axonal regeneration was assessed by tracing the axons of the injured optic nerve after injection of the Alexa555-conjugated CTB into the vitreous humor 12 days after crush injury. Results revealed that the axons of the optic nerve regenerated through transduction of TAT-Pep5, compared with TAT-control (Figures $4 \mathrm{~b}$ and c). Notably, some axons reached beyond the $1-\mathrm{mm}$ point distal to the crush site in the TAT-Pep5-treated group, whereas no axons 
a

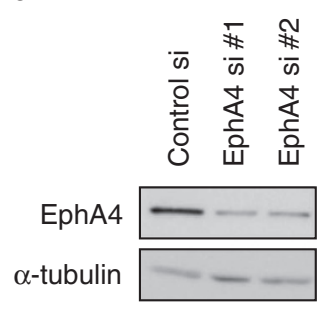

C

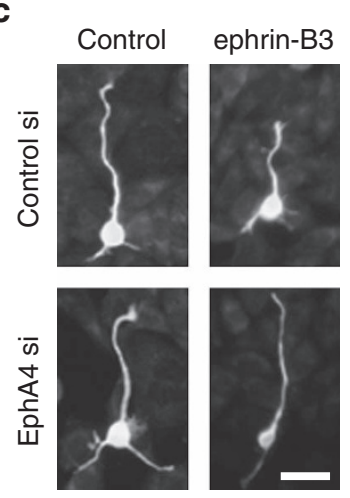

e

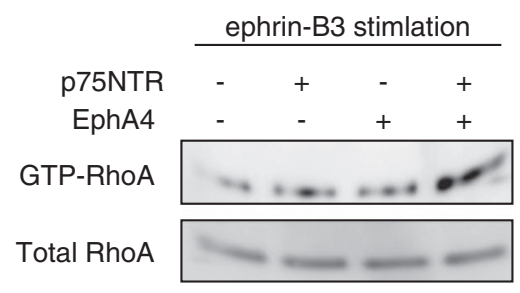

b

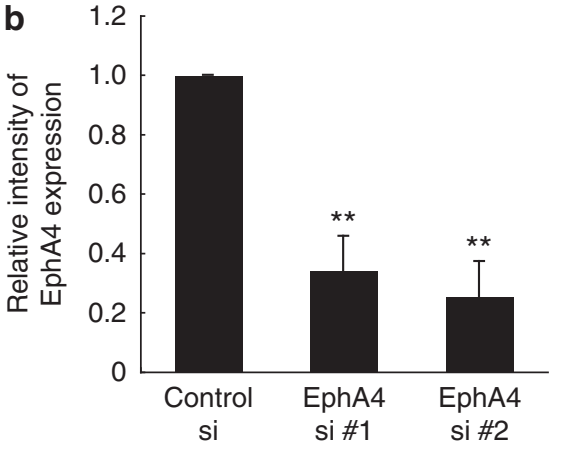

d

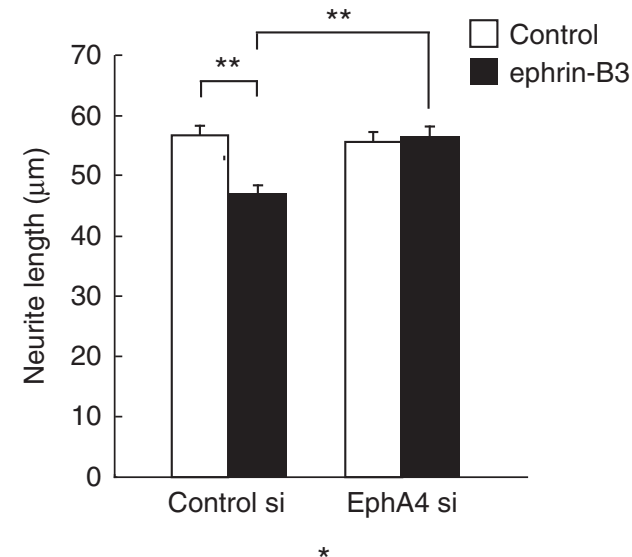

f

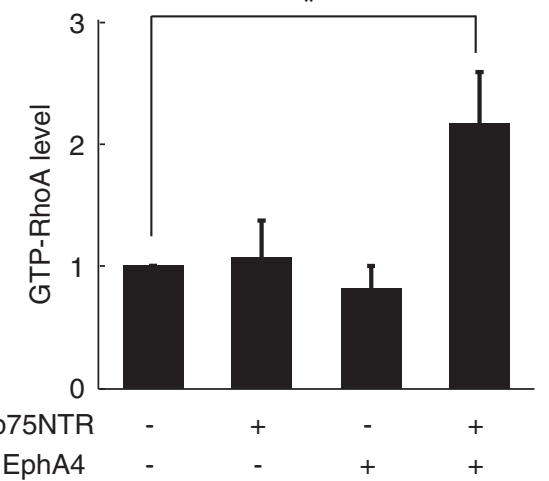

Figure 2 p75NTR is involved in neurite growth inhibition by ephrin-B3. (a) Western blots for EphA4 and $\alpha$-tubulin in EphA4 siRNA- and non-targeting siRNA-transfected cortical cells. (b) Quantitative data for (a) $(n=3)$. (c) The siRNA-transfected cortical neurons were cultured on control/ephrin-B3-CHO cells for $24 \mathrm{~h}$. Scale bar $=20 \mu \mathrm{m}$. (d) The graph illustrates mean neurite length $(n=711)$. EphA4 knockdown blocked neurite growth inhibition by ephrin-B3. (e) Pulldown assay for GTP-RhoA in ephrinB3-stimulated HEK293T cells. (f) Quantitative data for $(\mathbf{e})(n=3)$. Ephrin-B3 treatment activated RhoA only in the cells p75NTR and EphA4 were coexpressed. (b), (d); ${ }^{*} P<0.01$, Scheffé's F-test. (f); ${ }^{*} P<0.05$, Mann-Whitney's U-test

were visible at the same location in the TAT-control group (Figure 4c). Thus, silencing the axon growth inhibitory signal, mediated by P75NTR, contributed to the regeneration of injured axons in the optic nerve.

\section{Discussion}

The results of this study show that p75NTR is involved in neurite growth inhibition by ephrin-B3 in a manner similar to other guidance molecules, including EphA7, ephrin-A6, ephrin-B2, and sema-3A. ${ }^{9,13,14}$ In contrast, Benson et al. ${ }^{10}$ reported a lack of involvement of p75NTR in ephrin-B3induced neurite growth inhibition, as the ephrin-B3 effect was observed even in neurons from p75NTR-deficient mice. The results of the current experimental paradigm showed that Pep5 and p75NTR siRNA partially, but significantly, reversed the inhibitory effect of ephrin-B3 in cortical neurons. The extent of the relative contribution of p75NTR to EphA4 in the ephrin-B3 signals may depend on the cellular environment and assay methods. Differences with respect to the type and the age of the neurons and differences in the relative levels of p75NTR:EphA4 expression may determine the contribution of p75NTR to axon growth inhibition mediated by ephrin-B3.

In this study, knockdown of EphA4 completely abolished the inhibitory effect of ephrin-B3, suggesting that EphA4 is essential for ephrin-B3-induced neurite growth inhibition. 
a

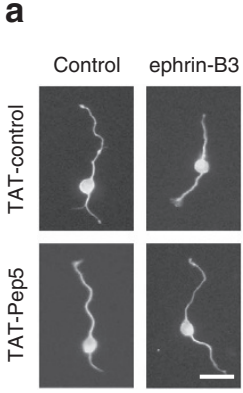

b

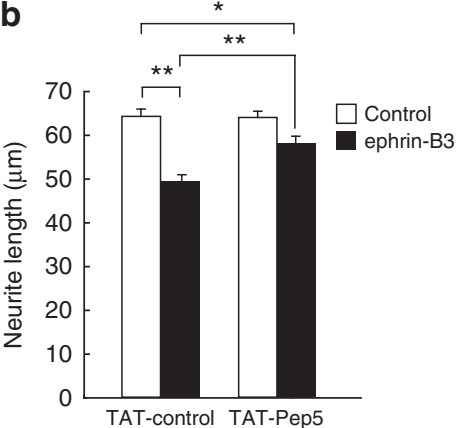

e

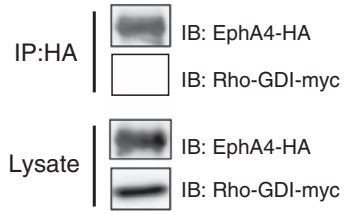

f
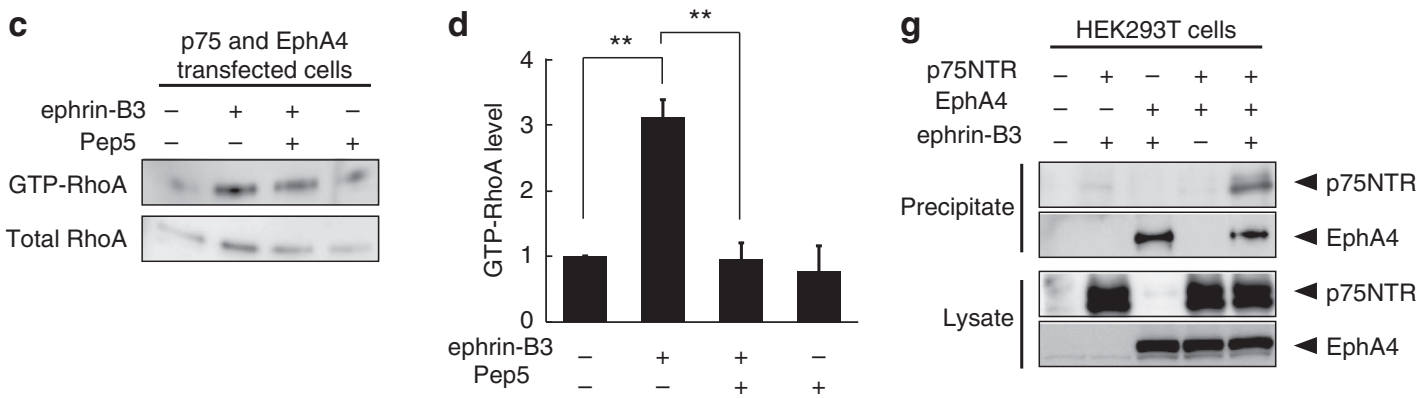

Figure 3 Ephrin-B3 inhibits neurite extension via p75NTR signaling. (a) The images demonstrate neuronal extension of the neurites in vitro, under the indicated conditions. Cortical neurons were cultured for $24 \mathrm{~h}$ on control/ephrin-B3-CHO cells in the presence or absence of $1 \mu \mathrm{M}$ TAT-Pep5. Scale bar $=20 \mu \mathrm{m}$. (b) The graph illustrates mean neurite length $(n=1075)$. Pep5 significantly attenuated the inhibitory effect of ephrin-B3. (c) Pulldown assay for GTP-RhoA in HEK293T cells expressing p75NTR and EphA4. Pep5 treatment attenuated RhoA activation induced by ephrin-B3. (d) Quantitative data for $(\mathbf{c})(n=3)(\mathbf{e})$. Immunoprecipitation of HA-tagged EphA4, followed by western blots for detection of myc-tagged Rho-GDI in the transfected HEK293T cells. Representative blots from three independent experiments. (f) Binding of ephrin-B3-Fc or BDNF to microwells coated with the p75NTR extracellular domain (ECD)-His tag or EphA4 ECD-His tag. Data plotted from three independent experiments. (g) Co-precipitation of p75NTR and ephrin-B3 using lysates prepared from HEK293T cells. HEK293T cells were transfected with p75NTR and/or EphA4 and were treated with ephrin-B3-Fc or control-Fc. Control-Fc and ephrin-B3/Fc were precipitated with protein G-Sepharose and subjected to SDS-PAGE. Representative blots from three independent experiments. (b), (d); ${ }^{*} P<0.05,{ }^{* *} P<0.01$, Scheffé's F-test

Furthermore, results demonstrated that ephrin-B3 does not bind to p75NTR directly and that EphA4 was required for the binding of ephrin-B3 to p75NTR. These findings prompted an assessment of the interaction between p75NTR and EphA4, but this study and others have been unable to demonstrate an interaction between endogenous p75NTR and either EphA4 (our unpublished data) or EphB2. ${ }^{9}$ However, an interaction of p75NTR with EphA4 in the transfected HEK293T cells was observed (data not shown).

As p75NTR is the bidirectional regulator of RhoA activity, simple deletion of p75NTR may not be a good method for induction of axonal regeneration in the CNS. Indeed, no significant axonal regeneration of the corticospinal tract was observed after spinal cord injury in p75NTR-deficient mice. 5,6 In the case of the optic nerve, previous studies have demonstrated a relatively low level of axonal regeneration after optic nerve crush in p75NTR-deficient mice, using the same method used in the present study. ${ }^{21}$ The extent of optic nerve regeneration was comparatively higher with the application of Pep5. This difference in effect may be dependent on neurotrophin expression. Some neurotrophins in the retina are upregulated after optic nerve injury ${ }^{19}$ and may act on retinal neurons through both Trk receptors and p75NTR. p75NTR promotes axonal elongation through neurotrophin binding and associates with Trk receptors to form a high-affinity ligand-binding site. Therefore, it is possible that the balance of opposing signals from neurotrophins and myelin-derived axonal growth inhibitors may determine the feasibility of optic nerve regeneration. Pep5 may be a promising agent that effectively inhibits axon growth inhibitory signals without affecting neurotrophin signaling.

\section{Materials and Methods}

Animals. C57BL/6 J mice were purchased from Japan SLC (Shizuoka, Japan). These mice were bred and maintained in the Institute of Experimental Animal Sciences at the Osaka University Graduate School of Medicine, with approval from the Institutional Animal Care and Use Committee for all experimental procedures (19-081-0). All mice were treated and cared for in accordance with Osaka University guidelines pertaining to the treatment of experimental animals.

Generation of ephrin-B3-expressing CHO. The Flp-in system (Life Technologies, Carlsbad, CA, USA) was used to generate ephrin-B3-expressing cells according to the manufacturer's recommendations. Total RNA was extracted from adult mouse spinal cord with Trizol (Life Technologies) and reversetranscribed using a High-Capacity cDNA Reverse Transcription Kit (Applied Biosystems, Foster City, CA, USA). An ephrin-B3 fragment containing a signal peptide and two restriction sites was generated and ligated into pcDNA5FRT (Life Technologies). This construct was transfected into Flp-in $\mathrm{CHO}$ cells, and stable expressing cells were generated after growth in medium containing $500 \mu \mathrm{g} / \mathrm{ml}$ Hygromycin B (Wako Pure Chemical Industries, Osaka, Japan) for 2 weeks. Expression of ephrin-B3 was confirmed by immunodetection using western blot. Control-CHO was generated through transfection of the empty vector into Flp-in $\mathrm{CHO}$ cells.

Western blot analysis. $\mathrm{CHO}$ cells and cortical cells were lysed on ice for $20 \mathrm{~min}$ with lysis buffer (10 mM Tris- $\mathrm{Hcl}(\mathrm{pH} 7.5), 150 \mathrm{mM} \mathrm{NaCl}, 1.0 \% \mathrm{NP}-40$, $25 \mu \mathrm{g} / \mathrm{ml}$ leupeptin, and $25 \mu \mathrm{g} / \mathrm{ml}$ aprotinin). The lysates were centrifuged at 
a

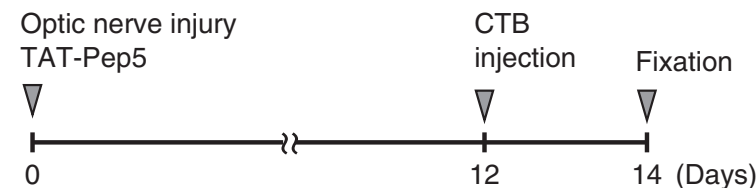

b

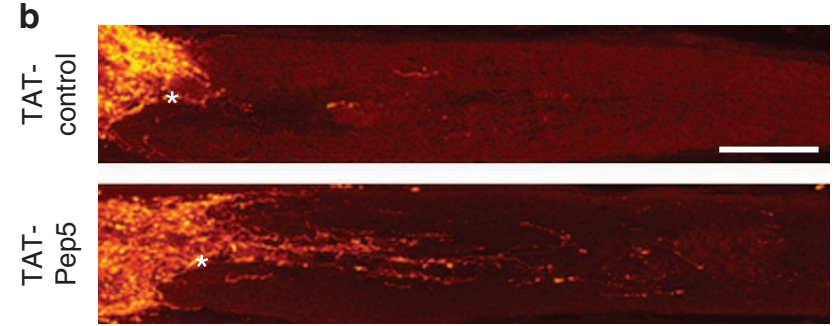

C

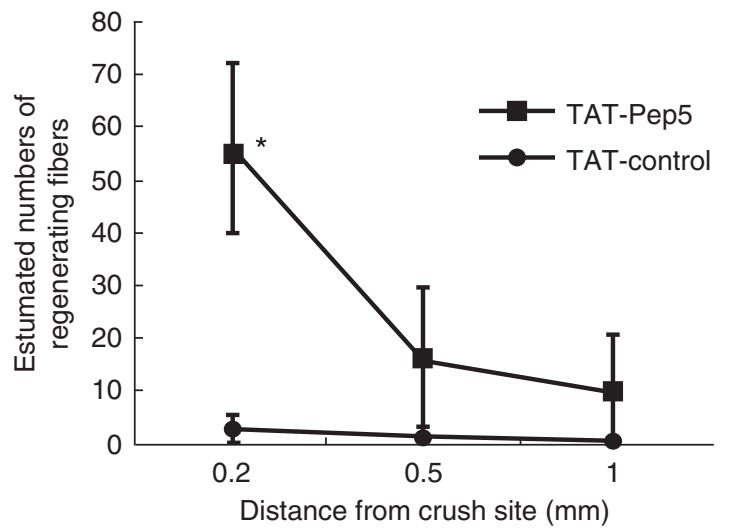

Figure 4 Pep5 induces axonal regeneration of the injured optic nerve. (a) Schedule of the experimental procedure. The optic nerves of P21 mice were crushed and TAT-Pep5 or TAT-Control was injected into the eye. On day 12 after crush, Alexa555-conjugated CTB was injected. On day 14 after crush, the optic nerves were fixed, and serial longitudinal sections were prepared. (b) Representative micrographs of longitudinal sections through the optic nerve showing that CTB-labeled axons extended distal to the injury site in TAT-Pep5treated mice, but not in TAT-control-treated mice. *Injury site. Scale bar $=200 \mu \mathrm{m}$. (c) Quantitative analysis of regenerating axons 14 days after injury (TAT-control, $n=3$; TAT-Pep5, $n=3$ ). ${ }^{*} P<0.05$, Mann-Whitney U-test

$17900 \times g$ at $4^{\circ} \mathrm{C}$ for $20 \mathrm{~min}$, the supernatants were collected and then boiled in sample buffer containing $\beta$-mercaptoethanol for $5 \mathrm{~min}$ and subjected to SDSPAGE. The proteins were transferred onto polyvinylidene difluoride membranes (EMD Millipore/Merck KGaA, Darmstadt, Germany) and incubated with a polyclonal anti-p75NTR antibody (1:1000; Promega, Firchburg, WI, USA), a polyclonal anti-ephrin-B3 antibody (1:1000; Life Technologies), a polyclonal antiEphA4 antibody (1:250; Santa Cruz Biotechnology, Santa Cruz, CA, USA), or a polyclonal anti- $\alpha$-tubulin antibody (1:1000; Santa Cruz Biotechnology). For detection, an ECL chemiluminescence system (GE Healthcare, Little Chalfont, UK) and HRP-conjugated secondary antibodies (1:1000; Cell Signaling Technology, Beverly, MA, USA) were used.

Neurite outgrowth assay. Cortical cells from mouse pups at postnatal days $0-1$ were dissociated through trypsinization $(0.25 \%$ trypsin in PBS for 15 min at $37^{\circ} \mathrm{C}$ ) followed by resuspension in serum-containing medium, trituration, and washing with medium three times. Cultures were grown in a $2 \%$ B27supplemented DME/F12 medium for $24 \mathrm{~h}$ on an ephrin-B3/Fc- or Fc-coated $35-$ $\mathrm{mm}$ dish (Greiner Bio-One, Kremsmünster, Austria). After plating the neurons, Y27632 (Calbiochem, San Diego, CA, USA), TAT-Pep5 (Calbiochem), or TATfused control peptide (TAT-GGWKWWPGIF, chemically synthesized by SigmaAldrich, St Louis, MO, USA) was added. For the co-culture assay, neurons were plated on a confluent monolayer of either ephrin-B3-CHO or control-CHO cells in a $35-\mathrm{mm}$ dish. Cells were fixed in $4 \%$ (wt/vol) paraformaldehyde and were immunostained with a monoclonal antibody recognizing Tuj 1 (1:1000; Covance, Princeton, NJ, USA). Then, the length of the longest neurite for each $\beta$ tubulin IIIpositive neuron was determined. Neurons were analyzed using an Olympus BX51 microscope (Olympus, Tokyo, Japan) and Image J software.

siRNA nucleofection. Cortical neurons were isolated and dissociated from E18 mice, as described above. The cells were washed and resuspended in room temperature Mouse Neuron Nucleofector Solution (Amaxa; Lonza Cologne AG, Cologne, Germany) at a final concentration of $5 \times 10^{6}$ cells $/ 100 \mu$ l. The cellnucleofector solution complex $(100 \mu l)$ and the various siRNA duplexes or control non-targeting siRNA $(500 \mathrm{pmol})$ were then gently mixed and transferred into a cuvette, followed by nucleofection using the nucleofector program 0-05. Immediately after electroporation, the cells were mixed with $500 \mu$ l of pre-warmed DMEM containing $10 \%$ FBS and the cell suspension was transferred onto poly-Llysine-coated dishes. The cells were placed in an incubator for $3 \mathrm{~h}$, after which the medium was replaced with fresh DMEM containing $10 \%$ FBS and the cells were incubated for an additional $72 \mathrm{~h}$. The cells were then subjected to western blotting, or were collected and plated for the neurite outgrowth assay.

Production of recombinant proteins. The p75NTR ECD and EphA4 ECD coding sequences were cloned into the pcDNA3.1 $(+)$ mammalian expression vectors (Life Technologies) to generate His-tag-fused proteins from HEK293T cells. Supernatants were collected and passed over Ni-NTA agarose beads (Qiagen, Venlo, Netherlands) and the beads were washed with denaturing buffer containing $20 \mathrm{mM}$ imidazole. Then $\mathrm{His}_{6}$-Proteins were eluted into a denaturing buffer containing $300 \mathrm{mM}$ imidazole. Fractions were pooled and concentrated (Amicon Ultra Centrifuge Tube; EMD Millipore/Merck) and stored at $-20^{\circ} \mathrm{C}$.

ELISA. ELISA was performed using 96-well microplates (Thermo Fisher Scientific, Waltham, MA, USA) that were coated with $0.1 \mu \mathrm{g} / \mathrm{ml}$ of p75NTR ECD-His or EphA4 ECD-His. The wells were blocked with $1 \% \mathrm{BSA}$ for $1 \mathrm{~h}$ at room temperature and then incubated with recombinant ephrin-B3/Fc (0 to $1 \mu \mathrm{g} / \mathrm{ml}$; R\&D Systems, Minneapolis, MN, USA) or BDNF (0 to $1 \mu \mathrm{g} / \mathrm{ml}$; Peprotech, Rocky Hill, NJ, USA). Two hours after incubation, the plates were washed and anti-ephrin-B3 antibody or anti-BDNF antibody (Promega) was added. HRP-conjugated secondary antibodies, the substrate reagent, and stop solution (R\&D Systems) were used to detect protein binding. Absorbance was measured at $450 \mathrm{~nm}$.

Affinity precipitation of GTP-RhoA. The transfected cells were stimulated with $10 \mu \mathrm{g} / \mathrm{ml}$ ephrin-B3/Fc for $5 \mathrm{~min}$. Ephrin-B3/Fc was preclustered by incubation with anti-human Fc (Sigma-Aldrich), a necessary step to activate the biological activity of ephrin Fc proteins. TAT-Pep5 or TAT-fused control peptide was added $30 \mathrm{~min}$ before the ephrin-B3 stimulation. The cells were lysed in a solution containing $50 \mathrm{mM}$ HEPES, PH7.4, 1\% Nonidet P-40,5\% glycerol, $150 \mathrm{mM}$ $\mathrm{Nacl}$, and $30 \mathrm{mM} \mathrm{Mgcl}$. The cell lysates were clarified by centrifugation $(17900 \times \mathrm{g})$ at $4{ }^{\circ} \mathrm{C}$ for $10 \mathrm{~min}$, and the supernatants were incubated with $50 \mu \mathrm{g}$ of Rho-binding domain of rhotekin beads at $4^{\circ} \mathrm{C}$ for $45 \mathrm{~min}$. The beads were washed three times with the lysis buffer and subjected to SDS-PAGE followed by immunoblotting with anti-RhoA antibody (Santa Cruz). The cell lysates were also immunoblotted for total RhoA. The levels of RhoA activation were calculated by comparing the band intensities of active RhoA bands with those of total RhoA in each lane using Multi Gauge software (Fuji Film Corporation, Tokyo, Japan). The values obtained were then divided by those of control, and the results were expressed as fold increases over the levels in the controls.

Co-precipitation and immunoprecipitation. Transfection experiments with p75NTR and/or EphA4 expression vectors for HEK293T cells were performed using Lipofectamine 2000 (Life Technologies), according to the manufacturer's protocol. The transfected cells were serum-starved overnight by incubation in DMEM without FBS. After washing the cells twice in PBS, cell lysis was performed using a lysis buffer. A co-precipitation assay was performed through treatment with $1 \mu \mathrm{g}$ of ephrin-B3/Fc or anti-HA tag antibodies for $3 \mathrm{~h}$ and protein G-Sepharose beads for $1 \mathrm{~h}$, under rotation at $4{ }^{\circ} \mathrm{C}$. After washing the beads three times with the lysis buffer, the proteins were eluted by boiling in sample buffer for $5 \mathrm{~min}$ and then subjected to SDS-PAGE, followed by western blotting.

Plasmid constructs and siRNA. Human p75NTR was subcloned into the pcDNA3.1 vector, as previously described (Yamashita et al., 2003). Human Rho-GDI was subcloned into pEF-BOS expression vector. Mouse EphA4 
constructs were provided by Dr. Rüdiger Klein. Mouse EphA4 siRNAs (Stealth siRNA; Life Technologies) were used for knockdown experiments. Non-targeting siRNA (Applied Biosystems, Tokyo, Japan) was used as a negative control to mouse EphA4 siRNA. Mouse p75NTR siRNAs were chemically synthesized (FASMAC, Kanagawa, Japan) and contained the following sequences: p75NTR \#1-sense (5'-GGAGACA UGUUCCACAGGCAUdTdT-3'), p75NTR \#1-antisense ( $5^{\prime}$-AUGCCUGUGGAACA UGUCUCCdTdT- $3^{\prime}$ ), ${ }^{22}$ p75NTR \#2-sense ( $5^{\prime}$-GCCUAUAUUGCUUUCAAGAGA-3'), p75NTR \#2-antisense (5'-UCUUGAAAGCAAUAUAGGCCA-3'). Scramble $\left(5^{\prime}\right.$-GC GCGCUUUGUAGGAUUCGdTdT-3' and 5'-CGAAUCCUACAAAGCGCGCdTdT-3') was used as a negative control to mouse p75NTR. ${ }^{22}$

Optic nerve injury and quantification of axonal regeneration. Optic nerve injury and quantification of axonal regeneration was performed, as described previously in detail. ${ }^{23}$ The left optic nerve of a postnatal day 21 mouse was exposed intraorbitally and crushed with fine forceps for $10 \mathrm{~s}, \sim 1 \mathrm{~mm}$ from the optic disc. Then, intravitreal injections of $1 \mu \mathrm{l}$ TAT-Pep5 $(15 \mu \mathrm{M})$ or TAT-control were performed with a pulled glass pipette affixed to a Hamilton syringe. Care was taken not to damage the lens. On day 12 after crush, $1 \mu$ Alexa555-conjugated cholera toxin beta subunit (CTB, $2 \mu \mathrm{g} / \mu \mathrm{l}$; Invitrogen) was injected into the vitreous with a glass needle. On day 14 after crush, the animals received an overdose of anesthesia followed by perfusion with $4 \%$ paraformaldehyde. The lens and the vitreous body were removed and the remaining eyecups with the nerve segment were post-fixed in $4 \%$ paraformaldehyde overnight at $4{ }^{\circ} \mathrm{C}$. The eyecups were then dehydrated in $15-30 \%$ sucrose overnight at $4{ }^{\circ} \mathrm{C}$ and immersed in Optimal Cutting Temperature compound (Tissue Tek, Sakura Finetechnical Co, Tokyo, Japan). Tissues were frozen in dry ice, serial cross sections $(16 \mu \mathrm{m})$ were prepared using a cryostat, and collected on MAS-coated glass slides. Images were acquired on a microscope (BX51; Olympus) equipped with a camera (DP71; Olympus) using the DP Controller software (version 3.1.1.267; Olympus). Axonal regeneration was quantified by counting the number of CTB-labeled axons extending $0.2,0.5$, and $1.0 \mathrm{~mm}$ from the end of the crush site in five different sections. The cross-sectional width of the nerve was measured at the point at which the counts were taken and was used to calculate the number of axons per millimeter of nerve width. $\Sigma$ ad, the total number of axons extending the distance $d$ in a nerve having a radius of $r$, was estimated by summing all the sections having a thickness $t(16 \mu \mathrm{m})$ where:

$\Sigma$ ad $=\pi r^{2} \times($ average axons $/ \mathrm{mm}$ width $) / t$

\section{Conflict of Interest}

The authors declare no conflict of interest.

Acknowledgements. This work was supported by CREST, JST, and a Grantin-Aid for JSPS Fellows (No. 235820) from the Japan Society for the Promotion of Science.

1. Filbin MT. Myelin-associated inhibitors of axonal regeneration in the adult mammalian CNS. Nat Rev Neurosci 2003; 4: 703-713.

2. Yamashita T, Tohyama M. The p75 receptor acts as a displacement factor that releases Rho from Rho-GDI. Nat Neurosci 2003; 6: 461-467.

3. Harrington AW, Li QM, Tep C, Park JB, He Z, Yoon SO. The role of Kalirin9 in p75/nogo receptor-mediated RhoA activation in cerebellar granule neurons. J Biol Chem 2008; 283 24690-24697.
4. Wang KC, Kim JA, Sivasankaran R, Segal R, He Z. p75 interacts with the Nogo receptor as a co-receptor for Nogo, MAG and OMgp. Nature 2002; 420: 74-78.

5. Song XY, Zhong JH, Wang X, Zhou XF. Suppression of p75NTR does not promote regeneration of injured spinal cord in mice. J Neurosci 2004; 24: 542-546.

6. Zheng B, Atwal J, Ho C, Case L, He XL, Garcia KC et al. Genetic deletion of the Nogo receptor does not reduce neurite inhibition in vitro or promote corticospinal tract regeneration in vivo. Proc Natl Acad Sci USA 2005; 102: 1205-1210.

7. Yamashita T, Tucker KL, Barde YA. Neurotrophin binding to the p75 receptor modulates Rho activity and axonal outgrowth. Neuron 1999; 24: 585-593.

8. Yamashita $T$, Higuchi $H$, Tohyama $M$. The $p 75$ receptor transduces the signal from myelinassociated glycoprotein to Rho. J Cell Biol 2002; 157: 565-570.

9. Naska S, Lin DC, Miller FD, Kaplan DR. p75NTR is an obligate receptor required for cues that cause sympathetic neuron growth cone collapse. Mol Cell Neurosci 2010; 45: $108-120$

10. Benson MD, Romero MI, Lush ME, Lu QR, Henkemeyer M, Parada LF. Ephrin-B3 is a myelin-based inhibitor of neurite outgrowth. Proc Natl Acad Sci USA 2005; 102 : 10694-10699.

11. Duffy $P$, Wang $X$, Seigel CS, Tu N, Henkemeyer M, Cafferty WB et al. Myelin-derived ephrinB3 restricts axonal regeneration and recovery after adult CNS injury. Proc Natl Acad Sci USA 2012; 109: 5063-5068.

12. Kullander K, Klein R. Mechanisms and functions of Eph and ephrin signaling. Nat Rev Mol Cell Biol 2002; 7: 475-486.

13. Lim YS, McLaughlin T, Sung TC, Santiago A, Lee KF, O'Leary DD. p75(NTR) mediates ephrin-A reverse signaling required for axon repulsion and mapping. Neuron 2008; 59: 746-758.

14. Poopalasundaram S, Marler KJ, Drescher U. EphrinA6 on chick retinal axons is a key component for p75(NTR)-dependent axon repulsion and TrkB-dependent axon branching. Mol Cell Neurosci 2011; 47: 131-136.

15. Dechant G, Barde YA. The neurotrophin receptor p75(NTR): novel functions and implications for diseases of the nervous system. Nat Neurosci 2002; 5: 1131-1136.

16. Bartsch U, Kirchhoff F, Schachner M. Immunohistological localization of the adhesion molecules L1, N-CAM, and MAG in the developing and adult optic nerve of mice. J Comp Neurol 1989; 284: 451-462.

17. Huber AB, Weinmann O, Brösamle C, Oertle T, Schwab ME. Patterns of Nogo mRNA and protein expression in the developing and adult rat and after CNS lesions. J Neurosci 2002; 22: 3553-3567.

18. Fu CT, Tran T, Sretavan D. Axonal/glial upregulation of EphB/ephrin-B signaling in mouse experimental ocular hypertension. Invest Ophthalmol Vis Sci 2010; 51: 991-1001.

19. Gao H, Qiao X, Hefti F, Hollyfield JG, Knusel B. Elevated mRNA expression of brainderived neurotrophic factor in retinal ganglion cell layer after optic nerve injury. Invest Ophthalmol Vis Sci 1997; 38: 1840-1847.

20. Hornberger MR, Dütting D, Ciossek T, Yamada T, Handwerker C, Lang S et al. Modulation of EphA receptor function by coexressed ephrinA ligands on retinal ganglion cell axons. Neuron 1999; 22: 731-742.

21. Fujita $Y$, Takashima R, Endo S, Takai T, Yamashita T. The p75 receptor mediates axon growth inhibition through an association with PIR-B. Cell Death Dis 2011; 2: e198.

22. Higuchi H, Yamashita T, Yoshikawa H, Tohyama M. Functional inhibition of the p75 receptor using a small interfering RNA. Biochem Biophys Res Commun 2003; 301: 804-809

23. Fuijta Y, Endo S, Takai T, Yamashita T. Myelin suppresses axon regeneration by PIR-B SHP-mediated inhibition of Trk activity. EMBO J 2011; 30: 1389-1401.

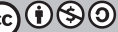

Cell Death and Disease is an open-access journal published by Nature Publishing Group. This work is licensed under a Creative Commons Attribution-NonCommercialShareAlike 3.0 Unported License. To view a copy of this license, visit http://creativecommons.org/licenses/by-nc-sa/3.0/ 